\title{
CÂNCER COLORRETAL NA POPULAÇÃO BRASILEIRA: TAXA DE MORTALIDADE NO PERÍODO DE 2005-2015
}

\author{
Colorectal cancer in the Brazilian population: mortality rate \\ in the 2005-2015 period \\ Cáncer colorrectal de la población brasileña: tasa de mortalidad \\ en el período 2005-2015
}

Artigo Original

\section{RESUMO}

Objetivo: Analisar a taxa de mortalidade por câncer colorretal no Brasil. Métodos: Trata-se de um estudo descritivo, retrospectivo, de série temporal e espacial, realizado no período de janeiro de 2005 a junho de 2015, com base nos dados do Sistema de Informação sobre Mortalidade. Para avaliar a tendência anual de óbitos por região, foi utilizado o teste de MannKendall, e regressões lineares para avaliar o sentido de crescimento. O nível de significância adotado foi de 5\%, sendo empregado o software R Core Team 2015. Resultados: A taxa de mortalidade no Brasil foi 7,98 óbitos/100 mil habitantes para o ano de 2014. Maiores taxas foram observadas nas regiões Sul e Sudeste. Quanto ao número de óbitos por sexo, prevaleceu o feminino em todas as regiões brasileiras, destacando-se a região Sudeste. Conclusão: $O$ estudo do câncer colorretal tem sua importância a nível epidemiológico por demonstrar um aumento crescente nos índices de mortalidade.

Descritores: Neoplasias Colorretais; Registros de Mortalidade; Mortalidade.

\section{ABSTRACT}

Objective: To analyze the colorectal cancer mortality rate in Brazil. Methods: Descriptive and retrospective spatial time series study conducted from January 2005 to June 2015 using data from the Mortality Information System. The annual trend of deaths by region was assessed using the Mann-Kendall test and linear regressions to evaluate the direction of the growth. The significance level was set at 5\% and the software R Core Team 2015 was used. Results: The mortality rate in Brazil was 7.98 deaths/100,000 inhabitants for the year 2014. Higher rates were observed in the South and Southeast regions. Regarding the number of deaths by sex, the female gender prevailed in all Brazilian regions, especially in the Southeast region. Conclusion: The study of colorectal cancer is epidemiologically important as it demonstrates a steady increase in mortality rates.

Descriptors: Colorectal Neoplasms; Mortality Registries; Mortality.

\section{RESUMEN}

Objetivo: Analizar la tasa de mortalidad por cáncer colorrectal en Brasil. Métodos: Se trata de un estudio descriptivo, retrospectivo, de serie temporal y espacial realizado entre enero de 2005 y junio de 2015 basado en datos del Sistema de Información sobre Mortalidad. Se utilizó la prueba de Mann-Kendall para valorar la tendencia anual de muertes por región y regresiones lineares para valorar el sentido de crecimiento. El nivel de significación adoptado fue del 5\% a través del software R Core Team 2015. Resultados: La tasa de mortalidad en Brasil fue de 7,98 muertes/100 mil habitantes en 2014. Se observaron tasas más elevadas en las regiones del Sur y Sudeste del país. Hubo prevalencia del sexo femenino para el número de muertes por sexo en todas las regiones brasileñas principalmente en la región Sudeste. Conclusión: El estudio del cáncer colorrectal es importante a nivel epidemiológico porque presenta un creciente aumento de los indices de mortalidad.

Descriptores: Neoplasias Colorrectales; Registros de Mortalidad; Mortalidad.

Recebido em: 06/02/2016 Revisado em: 04/04/2016 Aceito em: 03/05/2016 


\section{INTRODUÇÃO}

O câncer colorretal (CCR) é uma neoplasia que acomete os segmentos do intestino grosso (cólon, reto e ânus) ${ }^{(1)}$. A maioria dos estudos epidemiológicos identifica o CCR pela Classificação Internacional de Doença (CID-10), subdividindo-o em neoplasias de cólon (C18), da junção retossigmoide (C19), do reto $(\mathrm{C} 20)$ e do ânus $(\mathrm{C} 21)^{(2)}$. Considerado um problema de saúde pública no mundo, o câncer colorretal é identificado como o terceiro tipo de câncer mais comum a nível global, situando-se após os cânceres de pulmão e mama ${ }^{(2,3)}$. É a quarta causa de óbito por câncer no mundo ${ }^{(2)}$ e o segundo tipo de câncer mais comum nos países ocidentais ${ }^{(2,3)}$.

No Brasil, nas regiões Sul e Sudeste, o CCR é considerado a terceira causa de morte por câncer $^{(3)}$. As maiores taxas de incidência são observadas em países desenvolvidos, como Estados Unidos (EUA), Austrália, Nova Zelândia e países da Europa Ocidental (2), enquanto as menores taxas de incidência são encontradas na África, e taxas intermediárias, em países da América Latina ${ }^{(2)}$. Segundo os dados do Instituto Nacional do Câncer José Alencar Gomes da Silva (INCA), a estimativa de novos casos no Brasil para 2016 é de 34.280, sendo 16.660 em homens e $17.620 \mathrm{em}^{\text {mulheres }}{ }^{(4)}$.

A estimativa realizada para o ano de 2008 quanto ao número de óbitos por CCR foi de 608 mil óbitos no mundo ${ }^{(5,6)}$. Entretanto, tem-se observado um declínio na mortalidade nos países da Europa Ocidental e do Norte e nos Estados Unidos ${ }^{(6)}$. As taxas de mortalidade de CCR para o ano de 2008 na Europa Oriental foram de 20,3 óbitos para cada 100 mil habitantes homens, e 12,1 óbitos para 100 mil habitantes mulheres ${ }^{(5)}$. Os coeficientes de mortalidade na África e Ásia foram baixos em comparação com os da Europa, América do Norte, Nova Zelândia e Austrália( ${ }^{(5)}$. Na Ásia, as taxas mais elevadas foram observadas no Japão e na China ${ }^{5}$. No Brasil, as maiores taxas de mortalidade por CCR foram observadas nas regiões Sul e Sudeste, variando entre 8,0 e 10,7 óbitos para cada 100 mil habitantes, entre 1980 e $1997^{(7)}$. Para o ano de 2001, as taxas de mortalidade no sexo masculino variaram entre 1,98 óbitos para cada 100 mil habitantes, em Manaus, e 15,89 óbitos para cada 100 mil habitantes, em Porto Alegre ${ }^{(7)}$. No sexo feminino, as taxas variaram entre 3,21 óbitos para cada 100 mil habitantes, em Belém, e 16,40 óbitos para cada 100.0000, em Porto Alegre $^{(7)}$.

São fatores de risco para o desenvolvimento de câncer colorretal: histórico familiar de CCR; idade; dieta baseada em gorduras animais; baixa ingestão de frutas, vegetais e cereais integrais; etilismo e tabagismo; obesidade e sedentarismo. $\mathrm{O}$ alto consumo de peixes, o baixo consumo de carnes vermelhas processadas e a prática de exercícios físicos são fatores protetores ${ }^{(8)}$. Com relação à idade, o CCR é mais frequente entre idosos, principalmente após os $60 \operatorname{anos}^{(3)}$, embora possa surgir em qualquer idade, sendo mais agressivo quanto mais precoce for o seu surgimento $\mathrm{e}$, geralmente, relacionado à transmissão hereditária ${ }^{(3)}$. As doenças inflamatórias intestinais, em especial a retocolite ulcerativa, também se relacionam com $\mathrm{CCR}^{(3)}$. Os pólipos adenomatosos esporádicos, responsáveis por $90 \%$ de todos os casos de câncer de cólon e reto, são condições pré-neoplásicas, havendo transformação do epitélio do cólon normal em pólipo adenomatoso ${ }^{(9)}$. A progressão adenocarcionoma é lenta e dura em média dez anos, fazendo com que esse tipo de neoplasia seja o alvo ideal de programas de prevenção e rastreamento da população ${ }^{(3,9)}$.

O CCR geralmente é assintomático, entretanto, devem ser valorizados os sintomas e sinais de alerta, tais como: alteração do hábito intestinal, dor abdominal, sangue oculto e alterações nas fezes. Os menos comuns são presença de muco nas fezes, dor no baixo ventre, anemia, queda do estado geral, tumor abdominal palpável, obstrução intestinal aguda, fístulas colônicas e peritonite fecal por perfuração intestinal, entretanto, fazem parte do quadro clinico da doença $a^{(3,10)}$. É considerado um dos cânceres que mais respondem às medidas de prevenção (controle dos fatores de risco e o rastreamento precoce). A detecção e remoção dos pólipos através de exames de rastreio tornam-se um importante método de prevenção, sendo a colonoscopia o procedimento de escolha para triagem e tratamento dessas lesões ${ }^{(9)}$. Em pacientes com baixo risco de desenvolver CCR, o rastreio deve ser realizado com pesquisa anual de sangue oculto nas fezes, sigmoidoscopia flexível a cada cinco anos ou retossigmoidoscopia rígida a cada dois anos para indivíduos a partir dos 50 anos de idade. Os pacientes com alto risco de desenvolver CCR devem ser rastreados com colonoscopia a partir dos 40 anos de idade ${ }^{(3)}$.

O desconhecimento da população sobre a importância do rastreamento do CCR contribui para um índice reduzido de adesão a exames diagnósticos e para um aumento da sua incidência. Nos Estados Unidos, o número de óbitos por esse tipo de câncer tem diminuído nos últimos anos, devido ao desenvolvimento de um sistema de rastreamento efetivo e da realização de campanhas educativas sobre o tema ${ }^{(9,11)}$.

O diagnóstico é realizado através da biopsia durante a colonoscopia, e o estadiamento é realizado através do exame físico, das tomografias de tórax, abdome e pelve e dosagem do antígeno carcinoembrionário ${ }^{(12)}$. O tratamento do CCR varia de acordo com o estádio, podendo ser cirúrgico, quimioterápico ou radioterápico, ou até uma associação de dois tipos de tratamento ${ }^{(13)}$.

A prevenção do CCR é baseada no conceito de que o intervalo de tempo entre o início da lesão e a instalação do câncer é geralmente longo, o que permite a adoção de 
políticas públicas de rastreamento populacional. Ações populacionais voltadas para a promoção em saúde de uma reeducação alimentar e de estímulos a prática de atividade física podem ter impacto nos aspectos epidemiológico do CCR. Além disso, a detecção precoce e o tratamento das lesões precursoras ou do câncer em fase inicial levam à redução da prevalência e mortalidade da doença ${ }^{(2)}$.

Diante do exposto, o objetivo deste estudo foi analisar a taxa de mortalidade por câncer colorretal no Brasil.

\section{MÉTODOS}

Estudo descritivo, retrospectivo, de série temporal e espacial, realizado no período de janeiro de 2005 a junho de 2015. Considerou-se o número de óbitos por ano, o número de óbitos por sexo, a taxa de mortalidade segundo a região e o ano, o número de óbitos segundo as regiões brasileiras e as diferenças percentuais dos óbitos entre os anos de acordo com as regiões brasileiras. Definiu-se óbito por neoplasia colorretal aquele em que a doença constava como a causa básica da morte na declaração de óbito (DO). Foram considerados os códigos C18.0 a C21.0 da Classificação Internacional de Doenças, $10^{\mathrm{a}}$ revisão (CID-10), referentes à neoplasia maligna do cólon $(\mathrm{C} 18.0)$, neoplasia maligna da junção retossigmoide (C19.0), neoplasia maligna do reto (C20.0) e neoplasia maligna do ânus (C21.0).

Os dados da mortalidade foram obtidos com base nos arquivos de declarações de óbito não nominais provenientes no Sistema de Informação sobre Mortalidade (SIM) ${ }^{(14)}$ do Departamento de Informática do Sistema Único de Saúde (DATASUS) e nos dados populacionais para o cálculo dos indicadores de mortalidade, pelas estimativas e projeções anuais do Instituto Brasileiro de Geografia e Estatística $(\text { IBGE })^{(15)}$. Para a análise dos dados, foi calculada a taxa de mortalidade por neoplasia colorretal para cada $100 \mathrm{mil}$ habitantes no Brasil e nas regiões brasileiras, por ano. A mortalidade proporcional foi calculada até o ano de 2014, pois o número total de óbitos por ano somente estava disponível no SIM até junho de 2015.

A área investigada foi o território brasileiro, categorizado em cinco regiões (Norte, Nordeste, Sudeste, Sul e Centro-Oeste). O Brasil possui uma área de 8.515.767,049 $\mathrm{km}^{2}$ e teve uma população estimada para o ano de 2005 de 184.184.264 habitantes, e para o ano de 2014, de 202.768.562 habitantes ${ }^{(15)}$. Região Norte: 14.698.878 habitantes em 2005 e 17.231.027 habitantes em 2014; região Nordeste: 51.019 .091 habitantes em 2005 e 56.186.190 habitantes em 2014; região Sudeste: 78.472.017 habitantes em 2005 e 85.115.623 habitantes em 2014; região Sul: 26.973.511 habitantes em 2005 e 29.016 .114 habitantes em 2014; e região Centro-Oeste: 13.020 .767 habitantes em 2005 e 15.219 .608 habitantes em $2014^{(15)}$.
Os dados foram descritos por meio de gráficos, tais como linha, setores e barras verticais, com o auxílio do Excel 2013. Para avaliar se houve tendência nas somas anuais de mortes por câncer de cólon e/ou retossigmoide, reto e ânus por região, foi utilizado o teste de Mann-Kendall e, para avaliar o sentido de crescimento, foram ajustadas as regressões lineares. O nível de significância adotado foi de 5\%, e o software utilizado foi o R Core Team 2015.

O projeto de pesquisa não foi submetido ao Comitê de Ética, por utilizar dados públicos secundários.

\section{RESULTADOS}

Foram registrados no Sistema de Informação de Mortalidade (SIM) do Departamento de Informática do Sistema Único de Saúde (DATASUS) 136.492 óbitos por CCR entre janeiro de 2005 e junho de 2015 (Figura 1).

O número de óbitos por CCR segundo o sexo, entre janeiro de 2005 e junho de 2015, foi de 71.197 (52\%) óbitos no sexo feminino e 65.275 (48\%) óbitos no sexo masculino - 20 óbitos não foram notificados no Sistema de Informação sobre Mortalidade (SIM).

A taxa de mortalidade do câncer colorretal para cada 100 mil habitantes no Brasil e regiões brasileiras, segundo os dados fornecidos pelo Sistema de Informação de Mortalidade (SIM) do Departamento de Informática do Sistema Único de Saúde (DATASUS) e as estimativas dos dados populacionais do Instituto Brasileiro de Geografia e Estatística (IBGE) (Figura 2), em 2005, foi de 5,60 óbitos para cada 100 mil habitantes, e em 2014, 7,98 óbitos para cada 100 mil habitantes. Aplicado o teste de Mann-Kendall, houve uma tendência significativa $(1,000(<0,001))$. Através da regressão linear $\left(-534,1+0,27^{*} \mathrm{ANO}\right)$, confirmou-se que essa tendência foi de crescimento.

Ao analisar as regiões separadamente, observa-se na região Norte que a tendência também foi de crescimento, pelo teste de Mann-Kendall $(0,867 \quad(<0,001))$ e pela regressão linear $(-278,9+0,14 * \mathrm{ANO})$. A taxa de mortalidade para a região Norte em 2005 foi de 1,60 óbitos por 100 mil habitantes, e em 2014 foi de 2,87 óbitos por 100 mil habitantes, representando a maior taxa de mortalidade da região. A menor taxa de mortalidade da região Norte foi registrada em 2006, com 1,55 óbitos por 100 mil habitantes.

Na região Nordeste, a taxa de mortalidade em 2005 foi de 2,32 óbitos por 100 mil habitantes, representando a menor taxa de mortalidade dessa região, e em 2014 foi de 4,12 óbitos por 100 mil habitantes, representando a maior taxa de mortalidade. Pelo teste de Mann-Kendall $(1,000$ $(<0,001))$ e pela regressão linear $(-412,0+0,21 *$ ANO), foi observada uma tendência também de crescimento para região Nordeste. 


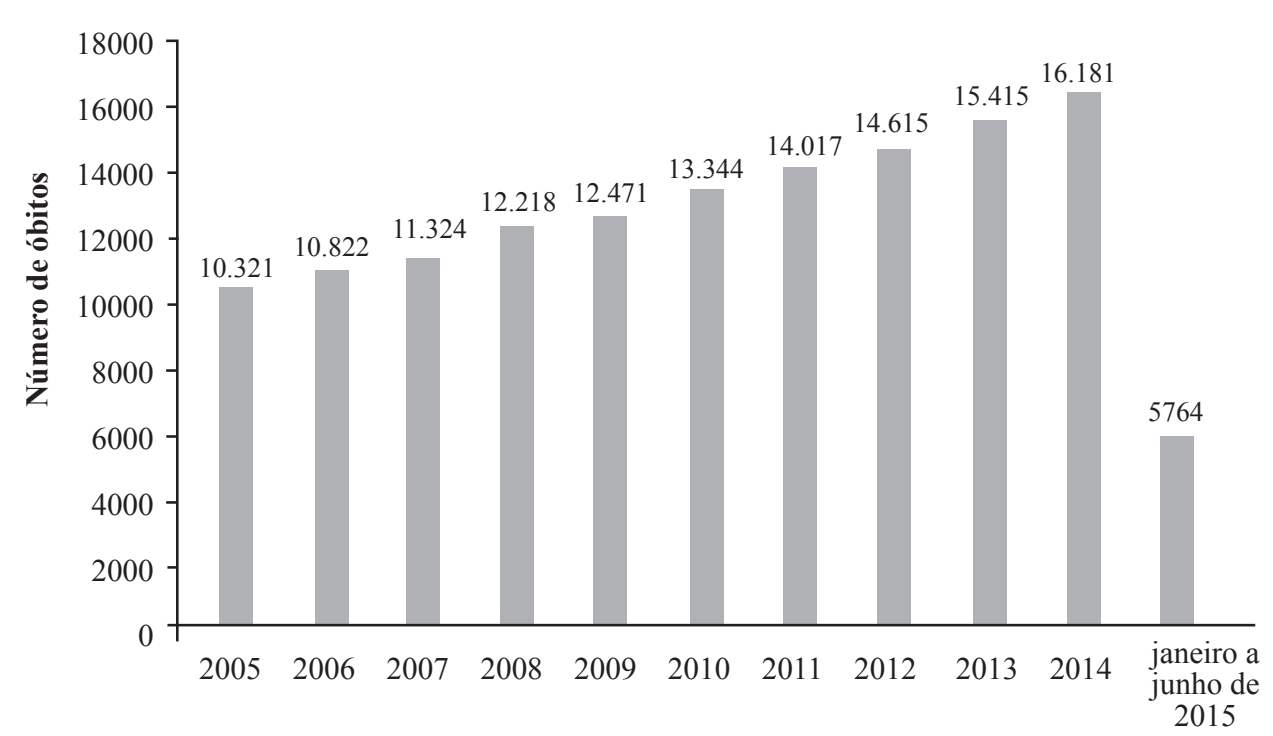

Figura 1 - Número total de óbitos por câncer colorretal (CCR) segundo o ano. Brasil, 2005 a 2015.

Fonte: Sistema de Informação de Mortalidade (SIM). Informática do Sistema Único de Saúde (DATASUS). Ano 2005-2015.

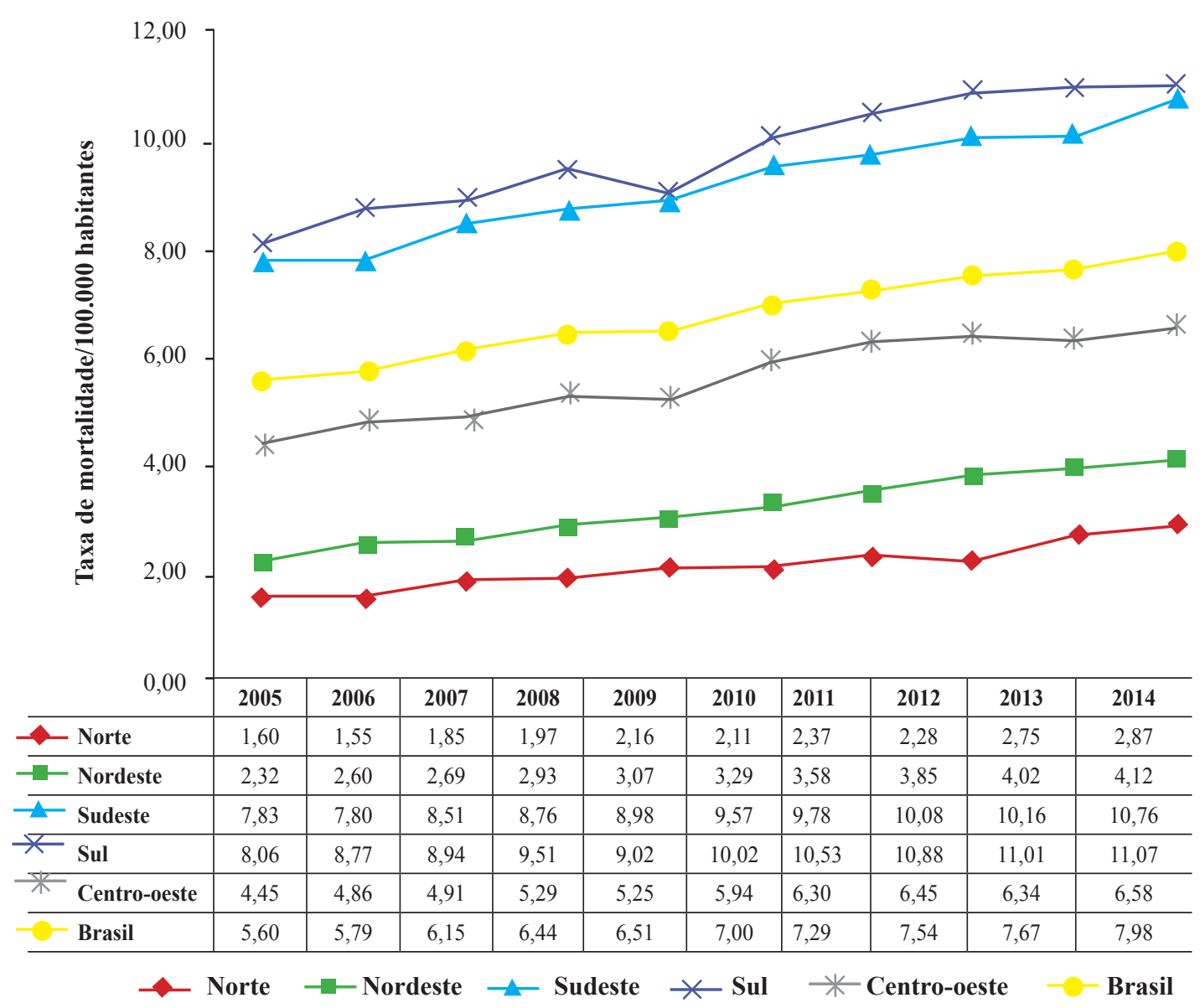

Figura 2 - Taxa de mortalidade por câncer colorretal (CCR) segundo região e ano. Brasil, 2005 a 2014.

Fonte: Sistema de Informação de Mortalidade (SIM). Informática do Sistema Único de Saúde (DATASUS). Instituto Brasileira de Geografia e Estatística (IBGE). Ano 2005-2014. 
Na região Sudeste, a taxa de mortalidade em 2005 foi de 7,83 óbitos por 100 mil habitantes, e em 2014 foi de 10,76 óbitos por 100 mil habitantes, representando a maior taxa de mortalidade para a região. A menor taxa de mortalidade para a região Sudeste foi registrada no ano de 2006 (7,80 óbitos por 100 mil habitantes). Através do teste Mann-Kendall $(0,956(<0,001))$ e da regressão linear $(-652,5+0,33 * A N O)$, também foi observada uma tendência de crescimento para a região Sudeste.

$\mathrm{Na}$ região Sul, a taxa de mortalidade em 2005 foi de 8,06 óbitos por 100 mil habitantes, representando a menor taxa de mortalidade registrada para a região, e em 2014 foi de 11,07 óbitos por 100 mil habitantes, representando a maior taxa de mortalidade registrada para a região. A tendência para essa região foi de crescimento, segundo o teste Mann-Kendall $(0,956(<0,001))$ e a regressão linear $(-679,3+0,34 * \mathrm{ANO})$.

Na região Centro-Oeste, a taxa de mortalidade foi de 4,45 óbitos por 100 mil habitantes em 2005, sendo a menor registrada, e de 6,58 óbitos por 100 mil habitantes em 2014, sendo a maior registrada. A tendência observada para a região Centro-oeste também foi de crescimento, segundo o teste Mann-Kendall $(0,911(<0,001))$ e a regressão linear $(-492,4+0,25 *$ ANO).

Destaca-se que a região Sul $(11,07)$ foi a que apresentou a maior taxa de mortalidade, seguida pelas regiões Sudeste $(10,76)$, Centro-Oeste $(6,58)$, Nordeste $(4,12)$ e Norte $(2,87)$.

Os registros do número de óbitos de acordo com as regiões brasileiras demonstraram que a região Sudeste manteve, ao longo do período observado, os maiores valores, totalizando 78.097 (57\%) óbitos, sendo seguida pela região Sul, 28.236 (21\%) óbitos, região Nordeste, 18.260 (13\%) óbitos, e região Centro-Oeste, 8.275 (6\%) óbitos. A região Norte apresentou os menores valores absolutos de mortalidade associada ao câncer colorretal, com 3.624 (3\%) óbitos (Figura 2).

Quanto à relação mortalidade por sexo (Figura 3), de acordo com as regiões, houve um maior número de óbitos no sexo feminino em todas elas. Na região Norte, observou-se $1.937(53,46 \%)$ óbitos no sexo feminino e 1.686 (46,53\%) óbitos no sexo masculino; na região Nordeste: 10.146 $(55,57 \%)$ óbitos no sexo feminino e 8.109 (44,42\%) no sexo masculino; na região Sudeste: 40.610 (52\%) óbitos no sexo feminino e $37.477(47,99 \%)$ óbitos no sexo masculino; na região Sul: 14.247 (50,46\%) óbitos no sexo feminino e 13.986 (49,53\%) óbitos no sexo masculino; e na região Centro-Oeste: $4.257(51,45 \%)$ óbitos no sexo feminino e 4.017 (48,54\%) óbitos no sexo masculino. A maior diferença percentual entre os sexos ocorreu no Nordeste; e a menor, no Sul.

Os dados revelaram diferenças percentuais dos óbitos por CCR entre os anos e as regiões brasileiras (Figura 4). Observou-se que na região Norte houve um crescimento de $25 \%$ de um ano para o outro (de 2012 para 2013), enquanto a região Sul apresentou a maior redução percentual nos coeficientes de mortalidade de um ano para o outro, ou seja, de $-4,4 \%$ entre 2008 e 2009. Maiores oscilações nos percentuais de óbitos ao longo dos anos foram observadas na região Norte, com valores que variaram de $-2,36 \%$ entre 2011 e 2012 e $25,54 \%$ entre 2012 e 2013 . Ao observar a linha referente à região Sudeste, ela oscilou menos do que as outras, sendo sempre crescente, ou seja, a tendência do Sudeste foi de crescimento, mas a uma taxa que não ultrapassou 6,8\% ao ano. Já as regiões Nordeste (13,25\% entre 2005 e 2006), Sul (10,17\% entre 2005 e 2006) e Centro-Oeste (14,25\% entre 2009 e 2010$)$ oscilaram a taxas muito maiores.

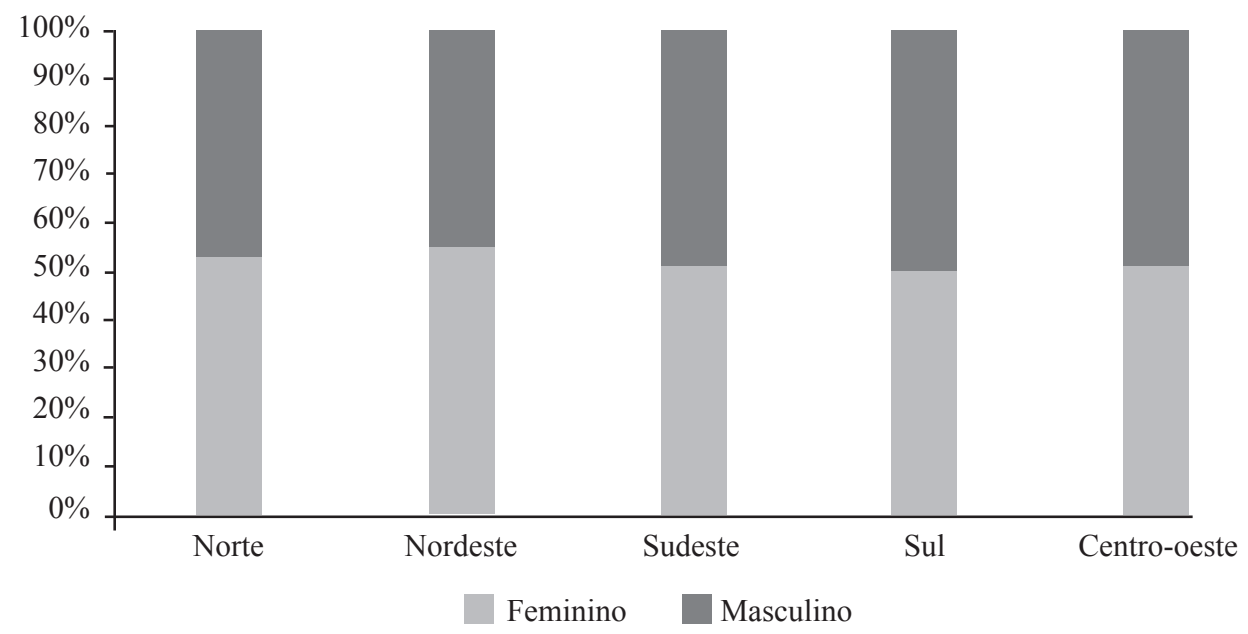

Figura 3 - Número total de óbitos segundo região e sexo. Brasil, 2005 a 2015.

Fonte: Sistema de Informação de Mortalidade (SIM). Informática do Sistema Único de Saúde (DATASUS). Ano 2005-2015. 


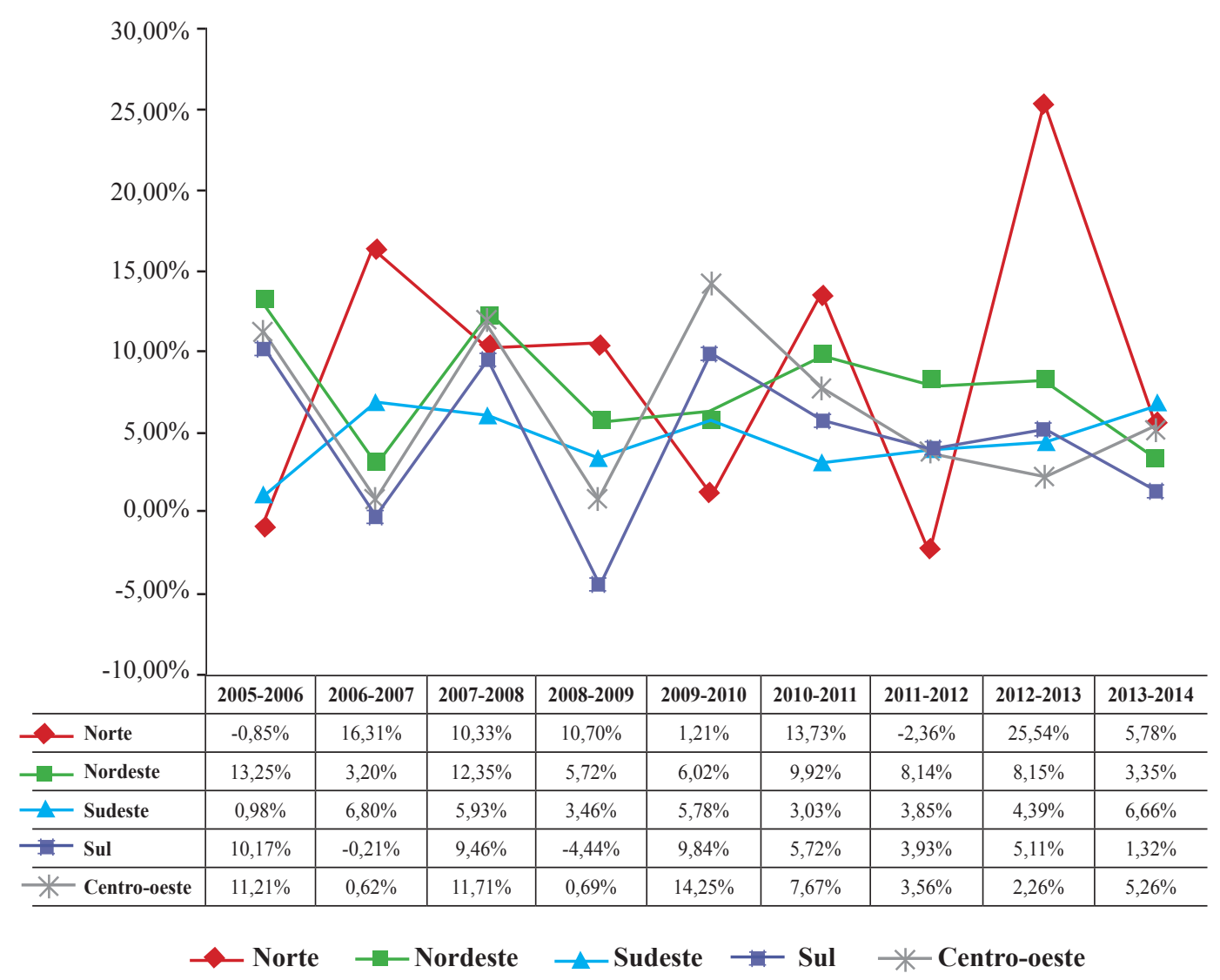

Figura 4 - Diferenças percentuais dos óbitos entre os anos por região. Brasil, 2005 a 2014.

Fonte: Sistema de Informação de Mortalidade (SIM). Informática do Sistema Único de Saúde (DATASUS). Ano 2005-2014.

\section{DISCUSSÃO}

O câncer colorretal (CCR) é considerado um problema de saúde pública mundialmente e diz respeito aos tumores malignos que afetam os segmentos do intestino grosso, como o cólon, o reto e o ânus ${ }^{(1)}$. Apesar dos avanços na medicina, principalmente em termos de diagnóstico e tratamento, a mortalidade causada por essa neoplasia é alta, de tal forma que sua sobrevida média global, em cinco anos, tem sido descrita como em torno de $55 \%$ nos países desenvolvidos e de $40 \%$ para países em desenvolvimento ${ }^{(16,17)}$.

Neste estudo, a análise do número de óbitos por CCR, por ano, mostrou que há um aumento crescente ao longo dos anos analisados, sendo o menor valor registrado no ano de 2005 e o maior, em 2014. O Brasil passa por um período de transição epidemiológica, em virtude da redução de óbitos por doenças infectocontagiosas e do aumento de doenças crônico-degenerativas, dentre elas, o câncer tem tido um lugar de destaque ${ }^{(18)}$. Isso se deve aos fatores de prevenção e às mudanças de hábitos de vida, respectivamente.

Dados da American Cancer Society, do ano de 2011, estimaram para os EUAcerca de 140 mil casos diagnosticados da doença e cerca de 50 mil óbitos por $\mathrm{CCR}^{(19)}$. Comparando com esses dados, para o mesmo período, foram registrados no Brasil 14.017 óbitos, entretanto, não há registro no SIM quanto ao número de casos diagnosticados no mesmo período, o que inviabiliza uma análise entre a incidência da doença e o número de óbitos.

A análise dos dados do SIM quanto o número de óbitos por CCR segundo o sexo demonstrou maior prevalência no sexo feminino. De acordo com dados do IBGE (2014) e do INCA (2014), o índice populacional brasileiro e a incidência da doença foram mais prevalentes no sexo feminino ao longo dos anos estudados ${ }^{(4,15)}$. Considera-se, então, que tanto a incidência da doença quanto a prevalência do número de óbitos, no Brasil, têm se mostrado mais expressivas no sexo feminino.

Neste estudo, a taxa de mortalidade refletiu um aumento crescente por CCR no Brasil. Em 2005, foram registrados 5,6 óbitos para cada 100 mil habitantes, enquanto em 2014 foram notificados 7,9 óbitos para cada 100 mil habitantes, dados que indicam uma diferença de 5.860 óbitos em números absolutos entre os anos de 2005 e 2014. Os maiores índices de taxas de mortalidade para CCR foram observados nas regiões Sul e Sudeste, e os menores índices, nas regiões Norte e Nordeste. Sabe-se que o CCR afeta 
principalmente a população dos países desenvolvidos ${ }^{(9)}$ e apresenta um risco de $5 \%$ a $6 \%$ de desenvolvimento dessa neoplasia ao longo da vida nos centros ocidentais ${ }^{(20)}$. Considerando que as regiões Sul e Sudeste são áreas mais desenvolvidas e industrializadas do Brasil, os aumentos da taxa de mortalidade e do número de óbitos nessas regiões podem estar relacionados aos fatores de risco presentes em países desenvolvidos. Essas diferenças geográficas podem ser atribuídas aos hábitos alimentares e a fatores ambientais que são impostos sobre um fundo de susceptibilidade genética $^{(21,22)}$.

$\mathrm{O}$ aumento nas taxas de mortalidade pode estar relacionado com os fatores de riscos associados a essa patologia, tais como idade maior que 50 anos, dieta com alto teor calórico, consumo de carnes vermelhas, sedentarismo, tabagismo e ingestão de álcool ${ }^{(9,12)}$. Pesquisa desenvolvida nos Estados Unidos demonstrou que o controle de fatores de risco passíveis de prevenção, como dieta e estilo de vida saudáveis, reduziu a incidência de CCR em 35\% naquele país ${ }^{(23)}$. Estimativas mostraram que $66 \%$ a $75 \%$ dos casos poderiam ser evitados com a adoção de estilos de vida mais saudáveis, dieta balanceada e prática de atividade física regular $^{(24)}$.

Quanto à idade, o câncer CCR é mais expressivo após os 50 anos, afetando menos de $10 \%$ a população abaixo dessa idade ${ }^{(3)}$. O crescimento da população idosa é um fenômeno mundial. No Brasil, a proporção de idosos aumentou de $9,1 \%$ em $1999^{(25)}$ para $11,7 \%$ em $2015^{(26)}$, e as projeções indicam que, em 2020, o Brasil será o sexto país do mundo em número de idosos ${ }^{(27)}$. Consequentemente, doenças relacionadas à idade, como o CCR, passam a ter índices sociodemográficos mais expressivos.

Ao analisar o número de óbitos segundo as regiões brasileiras, os dados revelaram que a região Sudeste obteve os maiores valores em números absolutos de óbitos ao longo dos anos analisados, seguida das regiões Sul, Nordeste, Centro-Oeste e Norte. Quando comparado à relação número de óbitos por sexo segundo as regiões, houve um maior número absoluto de óbitos no sexo feminino em todas elas, também com predomínio na região Sudeste. Uma possível explicação para o destaque da região Sudeste quanto ao maior número de óbitos por região e o maior número de óbitos por sexo feminino deve-se ao maior índice populacional e maior índice do sexo feminino nessa região(15).

Ao analisar as diferenças percentuais dos óbitos por CCR ao longo dos anos, segundo as regiões brasileiras, observaram-se diversas oscilações nos valores registrados. Destaca-se a maior redução percentual no número de óbitos na região Sul e a maior taxa de crescimento no número de óbitos na região Norte. A região que se revelou mais estável em relação à taxa de crescimento de óbitos foi a região Sudeste.
No Brasil, a cobertura do Sistema de Informação sobre Mortalidade (SIM) e a qualidade da informação das declarações de óbitos que alimentam o sistema variam conforme as diferentes regiões brasileiras ${ }^{(24)}$. Essas diferenças regionais podem superestimar ou subestimar os coeficientes de mortalidade, pois apresentam limitações quantitativas (sub-registro de óbitos e deficiências no fluxo das declarações) e limitações qualitativas (informações incorretas e erros no processamento de codificação da causa básica) ${ }^{(24,28)}$. Entretanto, nas declarações de óbitos em que a causa básica é uma neoplasia, pressupõe-se que as limitações qualitativas sejam minimizadas em função da natureza evolutiva da doença, a qual exige um tratamento hospitalar prolongado e exames complementares elucidativos ${ }^{(28)}$.

\section{CONCLUSÃO}

O estudo do câncer colorretal tem sua importância a nível epidemiológico, por demonstrar um aumento crescente nos índices de mortalidade, principalmente na população feminina e nas regiões mais desenvolvidas do território brasileiro. As oscilações demonstradas nas diferenças percentuais quanto ao número de óbitos por CCR requerem avaliações mais acuradas quanto à qualidade das informações das declarações de óbitos.

\section{REFERÊNCIAS}

1. Oliveira TR, Fortes RC. Hábitos alimentares de pacientes com câncer colorretal. J Health Sci Inst. 2013; 31(1):59-64.

2. Secretaria Municipal da Saúde (São Paulo), Coordenação de Epidemiologia e Informação. Boletim CEInfo Análise $n^{\circ}$ 06, Novembro/2012. São Paulo: Secretaria Municipal da Saúde; 2012. 35p.

3. Gomes CIMR, Furtado PCF, Silva CSF, Coelho M, Rocha DC, Coutinho FLS. Estudo sobre a acurácia da colonoscopia na detecção do câncer colorretal. Rev Méd Minas Gerais. 2013;23(3):307-10

4. Instituto Nacional de Câncer (INCA). Estimativa 2016. Incidência de Câncer no Brasil. [Internet]. Rio de Janeiro: INCA; 2016 [acesso em 2015 Dez 15]. Disponível em: http://www.inca.gov.br/ estimativa/2016/tabelaestados.asp?UF=BR

5. Xin KP, Du ML, Liu ZY, Wang WR, Qian YG, Liu L et al. Colorectal cancer mortality in Inner Mongolia between 2008 and 2012. World J Gastroenterol. 2014;20(25):8209-14

6. Shin A, Jung KW, Won YJ. Colorectal cancer mortality in Hong Kong of China, Japan, South Korea, and Singapore. World J Gastroenterol. 2013;19(7):979-83. 
7. Neves FJ, Mattos IE, Koifman RJ. Mortalidade por câncer de cólon e reto nas capitais brasileiras no período 1980-1997. Arq Gastroenterol. 2005;42(1):63-70

8. Ministério da Saúde (BR), Secretaria de Atenção à Saúde, Instituto Nacional de Câncer - INCA. Estimativas da incidência e mortalidade por câncer no Brasil. Rio de Janeiro: INCA; 2011.

9. Santos TP, Carvalho LPC, Souza ECR, Lise M. Conhecimento dos usuários do serviço público de saúde sobre câncer colorretal e sua prevenção. Rev AMRIGS. 2013;57(1):31-8

10. Lieberman DA, Weiss DG, Bond JH, Ahnen DJ, Garewal H, Harford WV, et al. Use of colonoscopy to screen asymptomatic adults for colorectal cancer. N Engl J Med. 2000; 343(3):162-8

11. Jemal A, Bray F, Center MM, Ferlay J, Ward E, Forman D. Global Cancer Statistics. Ca Cancer J Clin. 2011; 61(2):69-90.

12. Fernandes AS Jr, Lima AAP, Lima EM, Horta HL, Coutinho LFP, Martins SPS, et al. Sociedade Brasileira de Cancerologia. Diretrizes Clínicas na Saúde Suplementar. Câncer de Cólon: Tratamento quimioterápico. Associação Médica Brasileira, Agência Nacional de Saúde Suplementar [Internet]. 2011 Jan [acesso em 2016 Jan 6]. Disponível em: http://diretrizes.amb.org.br/ans/cancer_de_colontratamento_quimioterapico.pdf

13. Brasil. Portaria $n^{\circ} .958$, de 26 de setembro de 2014. Aprova as Diretrizes Diagnósticas e Terapêuticas do Câncer de Cólon e Reto. Ministério da Saúde: Secretaria de Atenção à Saúde. 2014. [acesso em 2016 Jan 6]. Disponível em: http://conitec.gov.br/images/ Artigos_Publicacoes/ddt_Colorretal_26092014.pdf

14. Ministério da Saúde (BR), Departamento de Informática do SUS (DATASUS). Sistema de Informação sobre Mortalidade [Internet]. Painel de Monitoramento da Mortalidade CID-10. [acesso em 2015 Dez 12]. Disponível em: http://svs.aids.gov.br/dashboard/ mortalidade/cid10.show.mtw

15. Instituto Brasileiro de Geografia e Estatística - IBGE. Estimativas de População Diretoria de Pesquisas, Coordenação de População e Indicadores Sociais [Internet]. 2014 [acesso em 2015 Dez 12]. Disponível em: http://ftp.ibge.gov.br/Estimativas_de_Populacao/ Estimativas_2014/serie_2001_2014_TCU.pdff.

16. Rego AGS, Borges ICV, Valença RJV, Teles JBM, Pinto LSS. Câncer Colorretal em Pacientes Jovens. Rev Bras Cancerol. 2012;58(2):173-80.

17. Instituto Nacional de Câncer José Alencar Gomes da Silva - INCA. Estimativa 2012: incidência de câncer no
Brasil [Internet]. Rio de Janeiro: INCA; 2011 [acesso em 2015 Dez 13]. Disponível em: http://www.inca.gov. br/estimativa/2012/estimativa20122111.pdf

18. Goldani MZ, Mosca PRF, Portela AK, Silveira PP, Silva $\mathrm{CH}$. O impacto da transição demográficoepidemiológica na saúde da criança e do adolescente do Brasil. Rev HCPA. 2012;32(1):49-57.

19. American Cancer Society. Colorectal cancer facts \& figures 2011-2013. Atlanta; 2011

20. Assis RVBF. Rastreamento e vigilância do câncer colorretal: guidelines mundiais. GED Gastroenterol Endosc Dig. 2011:30(2):62-74.

21. Ashktorab H, Nouraie M, Hosseinkhah F, Lee E, Rotimi C, Smoot D. A 50-year review of colorectal cancer in African Americans: implications for prevention and treatment. Dig Dis Sci. 2009;54(9):1985-90

22. Zandona B, Carvalho LP, Schimedt J, Koppe DC, Koshimizu RT, Mallamann ACM. Prevalência de adenomas colorretais em pacientes com história familiar para câncer colorretal. Rev Bras Coloproctol. 2011;31(2):147-54.

23. Richardson LC, Tai E, Rim SH, Joseph D, Plescia M. Vital Signs: colorectal cancer screening, incidence, and mortality. United States. Mortal Wkly Rep. 2011;60(26):884-9

24. Vasques ALR, Peres MA. Tendência temporal da mortalidade por câncer de cólon e reto em Santa Catarina no período entre 1980 a 2006. Epidemiol Serv Saúde. 2010;19(2):91-100.

25. Minayo MCS. O envelhecimento da população brasileira e os desafios para o setor saúde. Cad. Saúde Pública. 2012;28(2):208-9.

26. Viana ALA, Silva HP, Ferreira MP. Demografia das regiões de saúde brasileiras [Internet]. São Paulo: RESBR; 2015. (Nota técnica, $n^{\circ}$ 6) [citado 2016 jun]. Disponível em: http://www.resbr.net.br/wp-content/ uploads/2015/11/Novos-Caminhos-6.pdf

27. Veras R. Envelhecimento, demandas, desafios e inovações. Rev Saúde Pública 2009;43(3):548-54

28. Neves FJ, Koifman RJ, Mattos IE. Mortalidade por câncer de cólon e reto e consumo alimentar em capitais brasileiras selecionadas. Rev Bras Epidemiol. 2006;9(1):112-20.

\section{Endereço para correspondência:}

Camila Costa Santos de Menezes

Avenida Murilo Dantas, 1349

Bairro: Farolândia

CEP: 49032-490 - Aracaju - SE - Brasil

E-mail: camilacsm@hotmail.com 